\title{
Correction to: Evaluation of properties and specific energy consumption of spinifex-derived lignocellulose fibers produced using different mechanical processes
}

\author{
Katarzyna Kępa (D) - Céline M. Chaléat (D) Nasim Amiralian (D) \\ Warren Batchelor (i) - Lisbeth Grøndahl (i) - Darren J. Martin (1)
}

Published online: 4 February 2020

(C) Springer Nature B.V. 2020

\section{Correction to:}

Cellulose (2019) 26:6555-6569

https://doi.org/10.1007/s10570-019-02567-x

Due to an equipment error, the values for the energy consumption of samples which were subjected to the milling process (presented in Table 2) are incorrect in the original publication. The value for sample M-20 is $20.2 \mathrm{kWh} / \mathrm{kg}$ and for sample M-40 is $33.2 \mathrm{kWh} / \mathrm{kg}$.
Those corrections will respectively affect values for other "dual processed" samples, which have been extruded and then milled, i.e., X-M samples, as the energy of these is the sum of energies for combined extrusion and milling processes. Finally, as a conclusion for this aspect of the publication, it should be

The original article can be found online at https://doi.org/10.1007/s10570-019-02567-x.

K. Kępa · C. M. Chaléat · N. Amiralian · L. Grøndahl ·

D. J. Martin ( $\square)$

Australian Institute for Bioengineering and

Nanotechnology, The University of Queensland, Building

75, Corner of College and Cooper Road, Brisbane,

QLD 4072, Australia

e-mail: darren.martin@uq.edu.au

W. Batchelor

Department of Chemical Engineering, Bioresource Processing Research Institute of Australia, Monash

University, Melbourne, VIC 3800, Australia

L. Grøndahl

School of Chemistry and Molecular Biosciences, The University of Queensland, Building 68, Cooper Road,

Brisbane, QLD 4072, Australia 
Table 2 Mechanical and physical properties and energy consumption of handsheets produced with different processing methods

\begin{tabular}{lllllllll}
\hline Sample & $\begin{array}{l}\text { Tensile } \\
\text { Index } \\
\left(\mathrm{Nm} \mathrm{g}^{-1}\right)\end{array}$ & $\begin{array}{l}\text { Strain at } \\
\text { Break (\%) }\end{array}$ & $\begin{array}{l}\text { Tensile } \\
\text { Strength } \\
(\mathrm{MPa})\end{array}$ & $\begin{array}{l}\text { Density } \\
\left(\mathrm{g} \mathrm{cm}^{-3}\right)\end{array}$ & $\begin{array}{l}\text { Porosity } \\
(\%)\end{array}$ & $\begin{array}{l}\text { Sample } \\
\text { thickness } \\
(\mu \mathrm{m})\end{array}$ & $\begin{array}{l}\text { Crystallinity } \\
\text { Index }(\%)\end{array}$ & $\begin{array}{l}\text { Energy } \\
\text { Consumption } \\
\left(\mathrm{kWh} \mathrm{kg}^{-1}\right)^{\mathrm{d}}\end{array}$ \\
\hline $\mathrm{H}$ & $84 \pm 10$ & $3.1 \pm 0.7$ & $84 \pm 10$ & $1.00 \pm 0.02^{\mathrm{b}}$ & $31 \pm 2^{\mathrm{b}}$ & $85 \pm 3$ & $72^{\mathrm{a}}$ & 43.3 \\
$\mathrm{X}-1$ & $32 \pm 4^{\mathrm{c}}$ & $1.8 \pm 0.4^{\mathrm{c}}$ & $25 \pm 3^{\mathrm{c}}$ & $0.75 \pm 0.05^{\mathrm{b}}$ & $48 \pm 4^{\mathrm{b}}$ & $116 \pm 9$ & $74^{\mathrm{a}}$ & 0.6 \\
$\mathrm{X}-3$ & $40 \pm 2$ & $2.1 \pm 0.3$ & $32 \pm 2$ & $0.82 \pm 0.05^{\mathrm{b}}$ & $44 \pm 3^{\mathrm{b}}$ & $107 \pm 5$ & $74^{\mathrm{a}}$ & 1.3 \\
$\mathrm{X}-6$ & $40 \pm 3$ & $1.8 \pm 0.4$ & $36 \pm 3$ & $0.91 \pm 0.05^{\mathrm{c}}$ & $38 \pm 4^{\mathrm{c}}$ & $92 \pm 5$ & $72^{\mathrm{a}}$ & 2.9 \\
$\mathrm{X}-10$ & $46 \pm 8$ & $2.0 \pm 0.2$ & $40 \pm 7$ & $0.88 \pm 0.06^{\mathrm{c}}$ & $40 \pm 4^{\mathrm{c}}$ & $94 \pm 7$ & $73^{\mathrm{a}}$ & 5.0 \\
$\mathrm{X}-3-\mathrm{M}-20$ & $77 \pm 7$ & $3.2 \pm 0.8$ & $83 \pm 8$ & $1.09 \pm 0.05^{\mathrm{c}}$ & $25 \pm 4^{\mathrm{c}}$ & $79 \pm 4$ & $72^{\mathrm{a}}$ & 21.5 \\
$\mathrm{X}-6-\mathrm{M}-20$ & $81 \pm 5$ & $3.5 \pm 0.6$ & $90 \pm 6$ & $1.11 \pm 0.06^{\mathrm{c}}$ & $24 \pm 4^{\mathrm{c}}$ & $85 \pm 6$ & $70^{\mathrm{a}}$ & 23.1 \\
$\mathrm{X}-10-\mathrm{M}-20$ & $79 \pm 5$ & $3.3 \pm 0.5$ & $85 \pm 5$ & $1.08 \pm 0.05^{\mathrm{c}}$ & $26 \pm 3^{\mathrm{c}}$ & $88 \pm 3$ & $71^{\mathrm{a}}$ & 25.2 \\
M-20 & $80 \pm 4$ & $3.0 \pm 0.3$ & $80 \pm 4$ & $1.0 \pm 0.1^{\mathrm{c}}$ & $31 \pm 7^{\mathrm{c}}$ & $99 \pm 11$ & $71^{\mathrm{a}}$ & $20.2^{\mathrm{a}}$ \\
M-40 & $82 \pm 4$ & $2.8 \pm 0.5$ & $87 \pm 4$ & $1.07 \pm 0.09^{\mathrm{c}}$ & $26 \pm 6^{\mathrm{c}}$ & $80 \pm 7$ & $68^{\mathrm{a}}$ & $33.2^{\mathrm{a}}$ \\
\hline
\end{tabular}

Number of replicates for value, $n=\mathrm{a}: 2, \mathrm{~b}: 5 ; \mathrm{c}: 9, n=10$ otherwise

${ }^{\mathrm{d}}$ Value calculated based on a single experiment

noted that the milling process is less energy intensive compared to high-pressure homogenization.

The correct version of Table 2 is provided in this article.
Publisher's Note Springer Nature remains neutral with regard to jurisdictional claims in published maps and institutional affiliations. 